\section{Exosomes promote restoration after an experimental animal model of intracerebral hemorrhage}

Journal of Cerebral Blood Flow \& Metabolism

2018, Vol. 38(5) 767-779

(C) Author(s) 2017

Reprints and permissions: sagepub.co.uk/journalsPermissions.nav DOI: $10.1177 / 0271678 \times 17708917$ journals.sagepub.com/home/jcbfm (SAGE

\author{
Laura Otero-Ortega', Mari Carmen Gómez de Frutos'”, \\ Fernando Laso-García'**, Berta Rodríguez-Frutos', \\ Esperanza Medina-Gutiérrez', Juan Antonio López ${ }^{2}$, \\ Jesús Vázquez², Exuperio Díez-Tejedor' and \\ María Gutiérrez-Fernández'
}

\begin{abstract}
Exosomes are gaining importance because they show great promise in therapeutic applications for several diseases. Particularly in stroke, exosomes derived from mesenchymal stem cell (MSC) therapy work as paracrine effectors responsible for promoting neurovascular remodeling and functional recovery. Adult male rats were subjected to intracerebral hemorrhage $(\mathrm{ICH})$ by intrastriatal injection of collagenase type IV; $24 \mathrm{~h}$ after surgery, MSC-derived exosomes were administered through the tail vein. The rats were euthanized at 7 or 28 days after treatment. Functional evaluation, lesion size, fiber tract integrity, axonal sprouting and white matter repair markers, biodistribution of exosomes and secretome proteomics were analyzed. Dil-labeled exosomes were found in the brains of the ICH-treated group and in the liver, lung and spleen. Animals receiving treatment with exosomes showed significantly better results in terms of functional recovery, lesion size, fiber tract integrity, axonal sprouting and white matter repair markers compared with the control group 28 days after stroke. Proteomics analysis of the exosomes identified more than 2000 proteins that could be implicated in brain repair function. In conclusion, white matter integrity was partly restored by exosome administration mediated by molecular repair factors. Exosomes as a treatment could be a heterogeneous process by nature and presents many factors that can influence brain plasticity in an adaptable and versatile manner.
\end{abstract}

\title{
Keywords
}

Brain repair, exosome, intracerebral hemorrhage, subcortical stroke, white matter injury

Received 28 November 2016; Revised 3 April 2017; Accepted II April 2017

\section{Introduction}

After decades of research focused on the search for a treatment for cortical strokes in experimental models in which the gray matter is most affected, a few translational studies are beginning to highlight the importance of considering the white matter component after damage. ${ }^{1,2}$ The high frequency of this damage motivates the search for an effective therapy to enhance the mechanisms underlying the repair of damaged white matter (axons and myelin) after a stroke.

Stem cell therapy is emerging as a viable repair therapy for the treatment of hemorrhagic stroke not only with gray matter affection, but including white matter injury. ${ }^{3-5}$ One of the most striking findings is that stem
'Department of Neurology and Stroke Center, Neuroscience and Cerebrovascular Research Laboratory, La Paz University Hospital, Neuroscience Area of IdiPAZ Health Research Institute, Autonomous University of Madrid, Madrid, Spain

${ }^{2}$ Cardiovascular Proteomics Laboratory \& Proteomics Unit, National Center for Cardiovascular Research, CNIC, Madrid, Spain

*These authors contributed equally to this work.

\section{Corresponding author:}

María Gutiérrez-Fernández, Department of Neurology and Stroke. Neuroscience and Cerebrovascular Research Laboratory, La Paz University Hospital, IdiPAZ Health Research Institute, Autonoma University of Madrid, Paseo de la Castellana 26I, 28046, Madrid, Spain. Email: mgutierrezfernandez@salud.madrid.org; exuperio.diez@salud.madrid.org 
cells are not integrated into residing neural networks but act indirectly, promoting brain repair and functional recovery. ${ }^{6}$ Although the mechanisms by which stem cell therapy act are still unknown, recent evidence has suggested they might be related to long-distance cell-tocell communication by paracrine function through secretory factors in the extracellular environment. ${ }^{7}$

Extracellular vesicles, including exosomes, microvesicles and apoptotic bodies have become recognized as important in cellular communication (particularly in paracrine and endocrine signaling ${ }^{8}$ ) and might be responsible for the long-distance effects of stem cell therapy. Specifically, exosomes derived from stem cells have recently been suggested to mediate restorative stem cell effects. ${ }^{9}$ Exosomes carry a concentrated group of functional molecules (DNA, ribosomal RNA, circular RNA, long noncoding RNA, microRNA, proteins and lipids) that serve as intercellular communicators not only locally but also systemically. These molecules are involved in many biological processes, ${ }^{8,10}$ and are an interesting source of therapeutic applications in the field of regenerative medicine for neural disabilities including another type of stroke: cerebral infarct., ${ }^{71}$ In particular, extracellular vesicles induce long-term brain protection, promote gray matter repair and neurological recovery and they modulate the peripheral post-stroke immune response. ${ }^{7}$

All these observations suggest the importance of investigating whether the administration of mesenchymal stem cell (MSC)-derived exosomes in clinical settings might set the path for a novel and innovative therapeutic concept in stroke. Using an experimental animal model of subcortical hemorrhage, the present study investigates for the first time the therapeutic effects of the intravenous administration of exosomes, such as inducing functional recovery and promoting oligodendrogenesis and white matter fiber repair when axonal tract integrity has been compromised.

\section{Materials and methods}

\section{Ethics statement}

In this study, animal care and experimental procedures were strictly in accordance with the Guide for the Care and Use of Laboratory Animals, and the study was approved by the medical school of La Paz University Hospital's Ethics Committee for the Care and Use of Animals in Research, according to the Spanish and European Union (EU) rules (86/609/CEE and RD53/ 2013). This committee includes endpoint criteria. All the animals received a postsurgery injection of Meloxicam as analgesia, and we have a protocol including the daily supervision of animals. If any animals show pain symptoms, they again receive Meloxicam as analgesia. Experiments were reported according to all stroke therapy academic industry roundtable ${ }^{12,13}$ and ARRIVE ${ }^{14}$ guidelines in terms of randomization, blinding and statistical powering.

\section{Animals and surgery}

Adult male rats were subjected to an experimental model of intracerebral hemorrhage (ICH). Anesthesia was induced using an anesthetic chamber with $8 \%$ isoflurane in a $5-\mathrm{L} / \mathrm{min}$ oxygen flow and the animals were maintained using a face mask with $4 \%$ isoflurane in a 2-L/min oxygen flow. After intraperitoneal injection of meloxicam $(2 \mathrm{mg} / \mathrm{kg})$, the animals were placed in a stereotactic frame. Craniotomy was performed adjacent to the bregma, and intrastriatal white matter injury was induced by injection of $1 \mu \mathrm{L}$ saline containing $0.5 \mathrm{U}$ collagenase type IV (Sigma-Aldrich). Stereotaxic coordinates of the injection site with respect to the bregma were as follows: $0.04 \mathrm{~mm}$ posterior, $0.35 \mathrm{~mm}$ lateral, $0.6 \mathrm{~mm}$ ventral, as previously described. ${ }^{15}$

A total of 54 male Sprague-Dawley rats (8-9 weeks old, weighing $200-250 \mathrm{~g}$ ) were used in this study. The study groups were as follows: (I) sham-operation group $(\mathrm{n}=10)$; (II) $\mathrm{ICH}$ control: $\mathrm{ICH}+1 \mathrm{ml}$ i.v. saline $(\mathrm{n}=20)$; (III) ICH treated: $\mathrm{ICH}+100 \mu \mathrm{g}$ i.v. exosomes $(\mathrm{n}=20)$ (Figure 1). An additional four animals were used for biodistribution analysis, which were euthanized at $24 \mathrm{~h}$ after treatment.

Treatment was administered via the tail vein at $24 \mathrm{~h}$ after surgery. Within the groups, the rats were then randomly divided into two subgroups that were euthanized at 7 days or at 28 days $(n=10$ each). The allocation of each animal to an experimental group was performed on the basis of a simple random choice: throwing a die (less than/equal to three: control; more than three: treatment). One rat from the control group and one rat from the treated group were excluded from the study because they died during the magnetic resonance procedure.

\section{Cell culture protocol, isolation and exosome characterization}

MSCs obtained from allogeneic adipose tissue of Sprague-Dawley rats $(250-300 \mathrm{~g})$ were cultured. The adipose tissue was digested with collagenase (Sigma-Aldrich) and incubated at $37^{\circ} \mathrm{C}$ in $5 \% \mathrm{CO}_{2}$. On the third pass, the cell cultures were divided into two groups:

1. $1 \times 10^{5}$ MSCs for characterization. The MSCs were trypsinized and incubated for $20 \mathrm{~min}$ at $4^{\circ} \mathrm{C}$. The flow cytometry analysis of the CD $90+/ \mathrm{CD} 29+/$ CD45-/CD11b- cells was performed using an FACSCalibur flow cytometer and CellQuest Pro software (Becton Dickinson). 


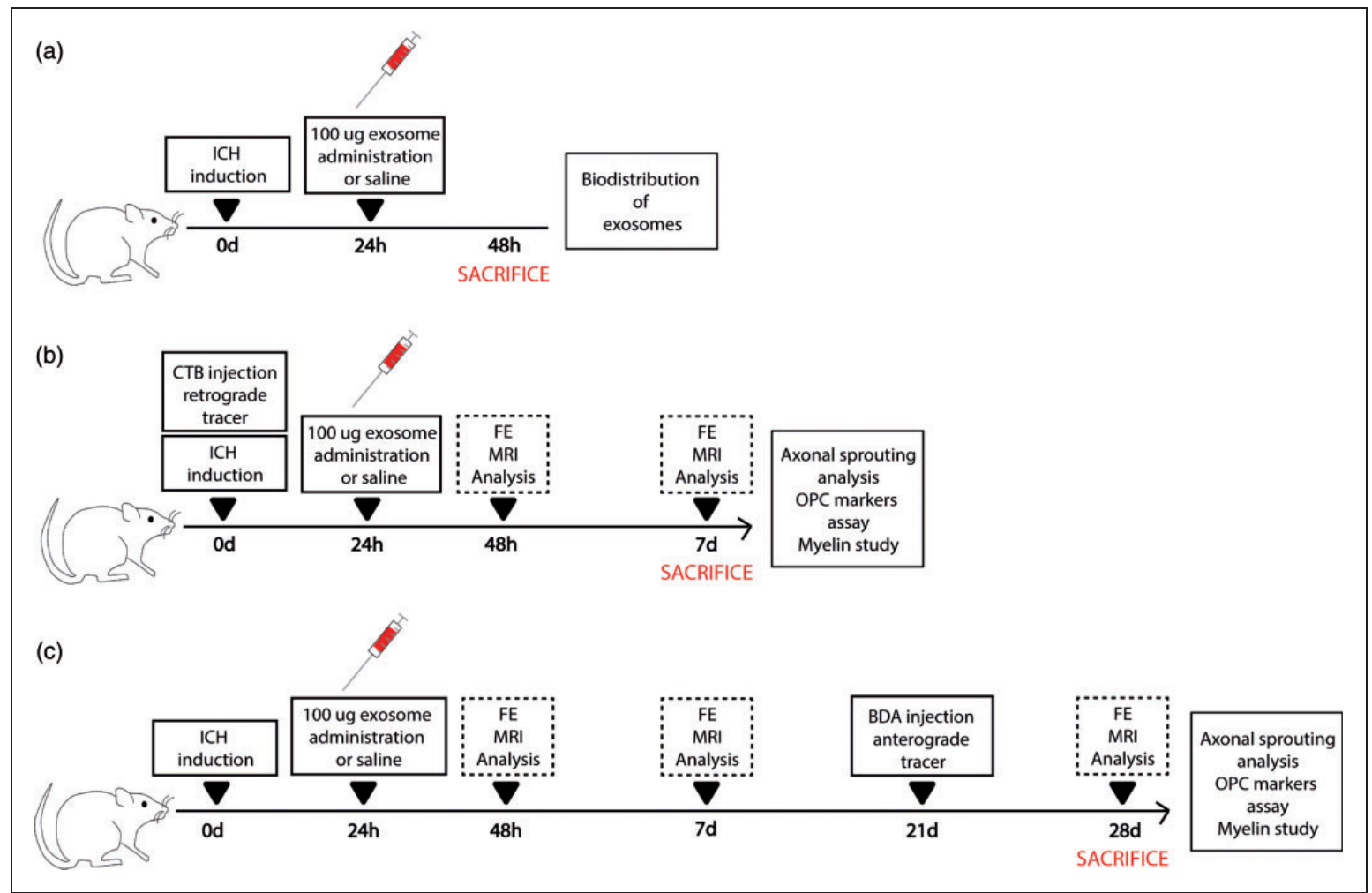

Figure I. Experimental protocol schematic. (a) Rats were subjected to an ICH by collagenase IV injection. Twenty-four hours later, rats received treatment (saline or exosomes). At $48 \mathrm{~h}$, histological studies for biodistribution of exosomes were performed. (b) Rats were injected with collagenase IV to induce hemorrhagic stroke. During the same surgery, animals were inoculated with CTB as retrograde tracer. At $24 \mathrm{~h}$ poststroke, treatment (saline or exosomes) was administered through the tail vein. Later, $48 \mathrm{~h}$ after ICH and on day 7, behavior and imaging studies were evaluated. Seven days after $\mathrm{ICH}$, histological studies were analyzed. (c) Rats were subjected to an ICH by collagenase IV and received treatment $24 \mathrm{~h}$ later (saline or exosomes). Forty-eight hours after ICH, on days 7 and 28 , behavior and imaging studies were evaluated. BDA was injected as anterograde tracers $2 \mathrm{I}$ days after ICH. Histological studies were analyzed 28 days after $\mathrm{ICH}$.

2. MSCs for exosome isolation. MSCs were cultured overnight with a free exosome-FBS culture medium. After $24 \mathrm{~h}$, the cell supernatants were collected and the exosomes were extracted using an exosome extraction kit (miRCURY ${ }^{\mathrm{TM}}$ Exosome Isolation Kit, Exiqon) following the manufacturer's instructions. Exosomes were used for treatment, for biodistribution study and for proteomic analysis.

Finally, the exosomes were characterized by studying morphology and size $(<100 \mathrm{~nm})$ using Nanosight and an electron microscope, and their phenotype expressing Alix and CD63 markers was examined by using immunofluorescence and Western blot techniques. ${ }^{16}$

\section{Biodistribution of exosomes}

For the evaluation of exosome biodistribution after treatment, four animals were used. The exosomes were labeled with DiI (Celltracker CM-DiI, Invitrogen) prior to administration. Cryosections ( $40 \mu \mathrm{m}$ thick) of the lung, liver, spleen and brain were counterstained with 4', 6-diamidino-2-phenylindole (DAPI) and biodistribution was analyzed by immunofluorescence staining at $24 \mathrm{~h}$.

To characterize which cell type contained the administered exosome in the brain, we did a co-staining with DiI-labeled exosomes and VEGF (1:500, Millipore), NeuN (1:100, Millipore), CNP-ase (1:500, SigmaAldrich) and Iba-1 (1:1000, Millipore), followed by the goat anti-mouse and anti-rabbit Alexa Fluor 488 antibody (1:750, Invitrogen). Images were acquired as a confocal maximum projection using a Leica TCS-SPE confocal microscope and the number of double-positive cells was counted in a minimum of 10 different microscopic fields using a $40 \times$ objective lens and ImageProPlus 4.1 software.

\section{Functional evaluation scales}

Functional evaluations were performed for all the animals by a blinded observer before surgery and after 
$48 \mathrm{~h}, 7$ days and 28 days. Motor performance was evaluated using the beam walking, Rogers and Rotarod tests. The Beam Walking test measured the ability of the rats to walk along a wooden beam $(2.5 \times 2.5 \times 80 \mathrm{~cm})$. Scores were assigned as follows: 0 , traversed the beam with no foot slip; 1 , traversed with grasping of the lateral side of the beam; 2, difficulty crawling along the beam but able to traverse; 3, required $>10 \mathrm{~s}$ to traverse the beam because of difficulty with walking; 4 , unable to traverse the beam; 5 , unable to move the body or any limb on the beam; and 6 , unable to stay on the beam for $>10 \mathrm{~s} .{ }^{17}$ The Rotarod test measured the time to fall from a rotating cylinder. ${ }^{5}$ A variant of Rogers' functional scale was used to assign scores as follows: 0 , no functional deficit; 1 , failure to extend forepaw fully; 2, decreased grip of forelimb while tail gently pulled; 3 , spontaneous movement in all directions, contralateral circling only if pulled by the tail; 4, circling; 5, walking only when stimulated; 6, unresponsive to stimulation with a depressed level of consciousness; and 7, dead. ${ }^{18}$ Animals had ever reached more than a score of 4 in the Rogers test. The researchers recording the data were entirely blinded to the experimental groups.

\section{In vivo magnetic resonance imaging}

Lesion size was analyzed after 7 and 28 days by magnetic resonance imaging (MRI) using a 7-Tesla horizontal bore magnet (Bruker Pharmascan, Ettlingen, Germany) and a T2-weight spin eco image. The lesion area was expressed as a percentage of the contralateral hemisphere, after correcting for brain edema. For the tractography analysis, diffusion tensor imaging (DTI) data were acquired with a spin echo single shot echo planar imaging (EPI) pulse sequence using the following parameters: TR/TE $8000 / 35 \mathrm{~ms}$; a signal average of 12 , a 30 noncollinear diffusion gradient scheme with a diffusion weighting $\mathrm{b}=500 \mathrm{~s} / \mathrm{mm}^{2}$ and $\mathrm{b}=1000 \mathrm{~s} / \mathrm{mm}^{2}$; 18 slices with a slice thickness of $1.5 \mathrm{~mm}$ without a gap; and a $35 \times 35 \mathrm{~mm}$ field of view. Total imaging time was $1 \mathrm{~h} 44 \mathrm{~min}$. All the EPI data were acquired with a single shot EPI sequence, $96 \times 96$ matrix, and zero filled-ink space to construct a $128 \times 128$ image matrix. Fractional anisotropy, mean diffusivity, trace, and the eigenvalues and eigenvector maps were calculated with a homemade software application written in Matlab (R2007a). The 3D fiber tract map was created using MedINRIA DTI Track software (http://www-sop.inia.fr/asclepios/ software/MedINRIA). DTI was performed at 7 and 28 days after treatment. The zoomed lesion site threedimensional diffusion tensor images were represented using ParaView 4.1.0 software as previously described. ${ }^{19}$ The researchers recording the data were entirely blinded to the experimental groups

\section{Mapping of motor cortex connections}

Changes in axonal sprouting after treatment were assessed using an intracortical injection of the anterograde neuronal tracer biotinylated dextran amine ([BDA] $10000 \mathrm{MW}$, Life Technologies, Grand Island, NY, USA). The rats' motor cortex was injected with BDA at 3 weeks after treatment in separate cohorts and they were euthanized one week after BDA injection (at 28 days after treatment). All the brains were fixed, frozen and coronally sectioned at $50 \mu \mathrm{m}$ and were stained with $\mathrm{Cy}$ 5-conjugated streptavidin (Jackson ImmunoResearch, West Grove, PA, USA). The sections were mounted, cover slipped and visualized under the Cy3 filter on a DMLB epifluorescent microscope (Leica Microsystems). The cortical cells labeled with BDA were manually quantified using neuroanatomical quantification software (StereoInvestigator, MBF Bioscience $)^{20}$ in four animals, 10 sections in each animal per group. Ipsilateral axons of the striatum and cortex were studied.

We used a retrograde neuronal tracer cholera toxin subunit B (CTB) (Cy3-labeled CTb, List Biological Laboratories, Campbell, CA, USA, diluted to $20 \mathrm{mg} / \mathrm{mL}$ in $10 \% \mathrm{DMSO}$ ) as another method to analyze changes in axonal sprouting after treatment. For mapping of CTB axons, $0.5 \mathrm{uL}$ of CTB (diluted to $20 \mathrm{mg} / \mathrm{mL}$ in $10 \%$ DMSO) was infused with collagenase at the time of stroke induction. The animals were euthanized $7 \mathrm{~d}$ after treatment, and the brains were fixed, frozen and coronally sectioned at $50 \mu \mathrm{m}$. The sections were mounted, cover slipped and visualized under the Cy3 filter on a DMLB epifluorescent microscope (Leica Microsystems). The cortical cells labeled with CTB were manually quantified using neuroanatomical quantification software (StereoInvestigator, MBF Bioscience) ${ }^{20}$ in four animals, 10 sections in each animal per group. Ipsilateral cortical cells were studied. The researchers recording the data were entirely blinded to the experimental groups.

\section{Immunofluorescence and western blot analyses}

Frozen sections were stained using the CryoMyelin Kit (Hitobiotech), which allows sensitive localization and visualization of myelin fibers. The mean intensity of myelin staining in the region of interest (ROI) was quantified using a Nikon Eclipse-Ti inverted microscope and NIS-elements software.

The lesion area was studied in detail using immunofluorescence and Western blot analyses, and the various antibodies used for these evaluations were CNPase (1:500, Sigma-Aldrich), A2B5 (1:500, Millipore) and MOG (1:100, Abcam), followed by goat anti-mouse and anti-rabbit Alexa Fluor 488 (1:750, Invitrogen). 
For the Western blot analysis, the units were normalized based on B-actin (1:400, Sigma-Aldrich). To quantify the expression of white matter-associated markers, the mean fluorescence intensity was evaluated using a $40 \times$ objective lens. The experiments, images and quantification of the samples were performed by blinded observers using the same microscope configurations to eliminate bias due to background normalization (4 animals for each group, 5 sections in each animal per group, 10 different microscope fields). The researchers recording the data were entirely blinded to the experimental groups.

\section{Proteomics data analysis}

For the proteomics analysis of exosome content, proteins were digested using the filter-aided sample preparation protocol. ${ }^{21}$ Peptides were loaded into the LC-MS/MS system for online desalting onto C18 cartridges and were analyzed by LC-MS/MS using a C-18 reversed phase nano-column $(75 \mu \mathrm{m}$ I.D. $\times 50 \mathrm{~cm}, 2 \mu \mathrm{m}$ particle size, Acclaim PepMap RSLC, 100 C18; Thermo Fisher Scientific, Waltham, MA, USA). A flow rate of $200 \mathrm{~nL} / \mathrm{min}$ was used to elute peptides from the RP nano-column to an emitter nanospray needle for real-time ionization and peptide fragmentation on an Orbitrap Elite mass spectrometer (Thermo Fisher). An enhanced FT-resolution spectrum (resolution $=35,000$ ) followed by the MS/MS spectra from the most intense 15 parent ions was analyzed along the chromatographic run. Dynamic exclusion was set at $30 \mathrm{~s}$.

For peptide identification, all the spectra were analyzed with Proteome Discoverer (version 1.4.0.29, Thermo Fisher Scientific) using SEQUEST-HT (Thermo Fisher Scientific). For database searching, the false discovery rate was calculated using inverted databases and the refined method. ${ }^{22}$ For the study of the biological functions of identified proteins, a gene ontology analysis was performed using the Gene Ontology enRIchment anaLysis and visuaLizAtion (GOrilla) research tool. ${ }^{23}$

\section{Statistical analysis}

The results were expressed as mean \pm standard error and the data were compared using the Kruskal-Wallis test followed by the Mann-Whitney test. The Beam Walking and Rogers test data are categorical; thus, the data have been presented as median and interquartile range. $\mathrm{T} 2$ and Tractography data were expressed as mean \pm standard deviation. Values of $p<.05$ were considered significant at a $95 \%$ confidence interval; the data were calculated using statistical SPSS 16 and GraphPad software. The power analysis showed that with nonparametric testing, at least 10 animals needed to be randomized to each group for a significance level of $5 \%$ (alpha) and a potency of $80 \%$ (1-beta).

\section{Results}

\section{Exosome characterization and biodistribution}

Regarding exosome characterization, we used four techniques: NanoSight, electron microscope, immunofluorescence and Western blot. The exosomes showed typical morphology and size $(<100 \mathrm{~nm})$ using NanoSight (Figure 2(a)) and an electron microscope (Figure 2(b)). Their phenotype was CD-63 (specific marker of exosome) as shown by immunofluorescence (Figure 2(c)) and Alix for the Western blot technique (Figure 2(d)).

Regarding biodistribution of the DiI-labeled exosomes, we analyzed histological sections of various organs. We found the DiI marker not only in the brain tissue but also in the peripheral organs such as the lung, liver and spleen at $24 \mathrm{~h}$ after administration (Figure 2(e)). We also found co-labeling between DiI and VEGF, NeuN, CNP-ase and Iba-1 at $24 \mathrm{~h}$ after exosome administration (Figure 2(f)).

\section{Effect of exosome treatment on functional recovery}

No significant differences were found in the functional outcome of the treated and control animals at $24 \mathrm{~h}$ and 7 days after treatment $(p>.05)$. However, compared with the control group, the treated animals showed significantly better beam walking $(1.00 \pm 0.94$ vs. $1.90 \pm 0.74$ points of the score, respectively; $p=.03)$, Modified Rogers' test $(0.39 \pm 0.67$ vs. $1.50 \pm 0.85$ points of the score, respectively; $p=.003$ ) and Rotarod test performances $(98.50 \pm 23.43$ vs. $76.30 \pm 24.66$ seconds, respectively; $p=.05) 28$ days after exosome treatment (Figure 3(a)).

\section{Effect of exosome treatment on lesion size and tract connectivity}

The lesion size viewed on MRI in the exosome-treated animals was indistinguishable from the control group at seven days $(14.42 \% \pm 3.65 \%$ vs. $14.05 \% \pm 0.45 \%)$. At 28 days, we observed significant differences between the ICHtreated animals $(9.08 \% \pm 0.72 \%)$ compared with the ICH controls $(10.60 \% \pm 0.77 \%)(p=.028)$ (Figure 3(b)).

The DTI tractography data showed similar results in mean axial diffusivity in both the controls and the treated groups 7 days after stroke. However, compared with the control animals, those rats receiving treatment with exosomes showed significantly improved mean axial diffusivity $(171.00 \pm 22.43$ vs. $128.67 \pm 26.08$, respectively; $p=.05$ ) at 28 days after treatment (Figure 3(c)). 


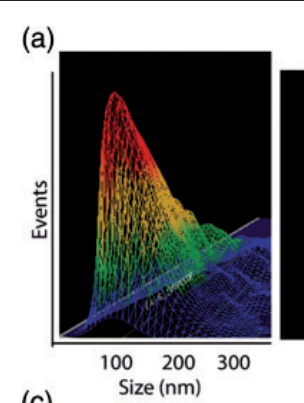

(c)

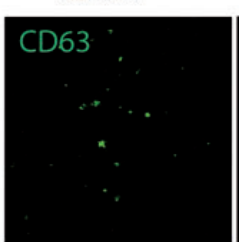

(e)
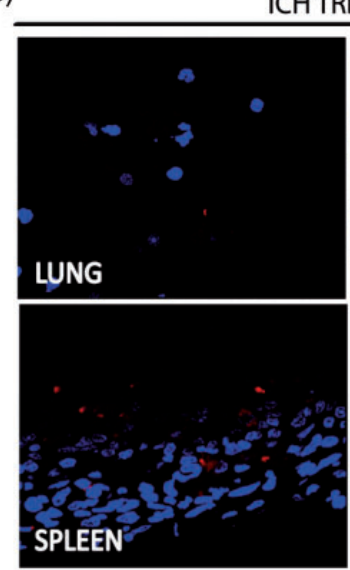
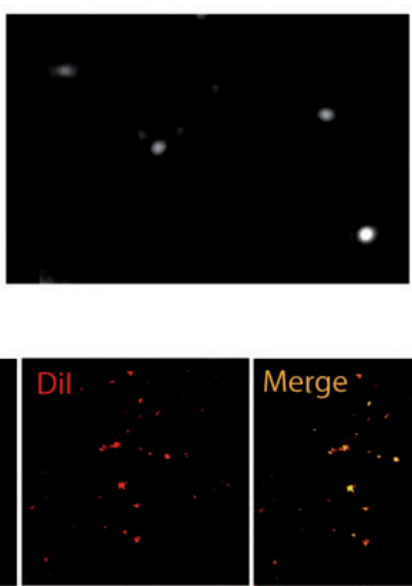

ICH TREATED
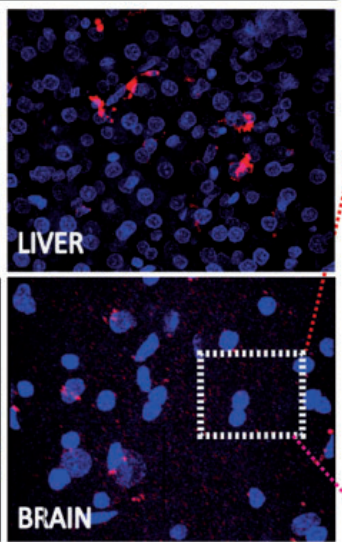

(b)

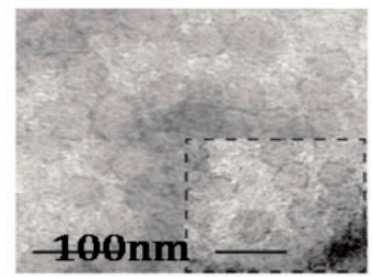

(d)
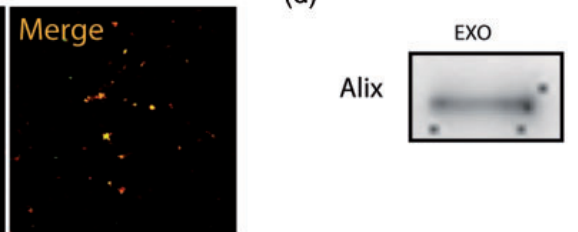

$96 \mathrm{kD}$

(f)

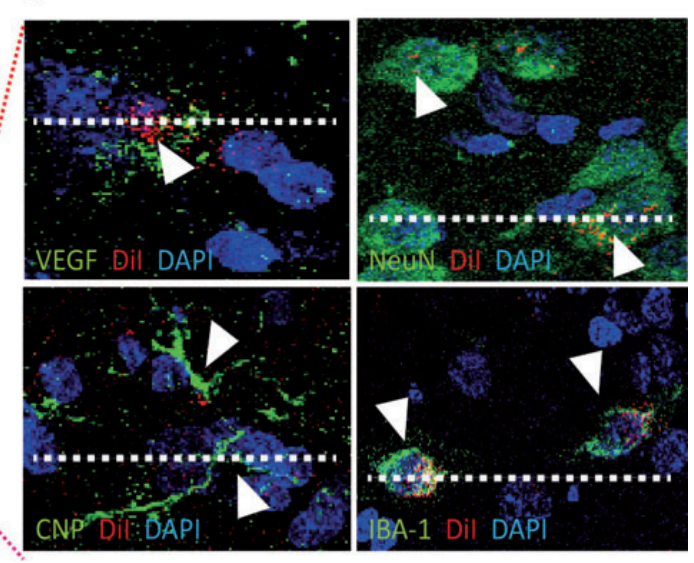

Figure 2. Exosome characterization and biodistribution. Exosomes were characterized using various techniques: (a) NanoSight, (b) electron microscope, (c) immunofluorescence and (d) Western blot. (e) Dil-exosomes were observed by immunofluorescence (red) after i.v. administration into the lung, liver, spleen and brain. (f) Dil co-localization was shown with VEGF, NeuN, CNP-ase and Iba-I in brain samples. (Data are shown as mean \pm SEM, $* p<.05 ; n=4$ animals per group).

\section{Axonal sprouting after exosome treatment}

ICH was produced in the subcortical white matter of the striatum. This region contains fascicles of axons projecting from the overlying cortex. To map the connections that are damaged by stroke, the neuroanatomical tracer CTB was co-injected with collagenase IV. Neurons retrogradely labeled with CTB injection into subcortical white matter represent cells with damaged axons at the stroke site. CTB-labeled neurons are present in overlying cortical areas ipsilateral and contralateral to the stroke site. There was no significant difference in the number of CTB-marker neurons between the control and treated groups, which could indicate that the severity of white matter injury is similar in both groups (Figure 3(d)).

Changes in axonal sprouting after treatment using intracortical injection of anterograde neuronal tracer with BDA were also analyzed. Motor cortical projections have an important role in motor function and movement. The anatomical tracer BDA was injected into the forelimb motor cortex 3 weeks after stroke, and the striatal axonal density was studied one week after the injection. A statistically significant high number of BDA-labeled axons were shown in the striatum in the exosome-treated group compared with the control animals $(483.95 \pm 61.21$ vs. $109.02 \pm 9.34$, respectively; $p<.05)$. These results could indicate that exosome treatment produces a significant axonal sprouting response from the ipsilateral cortex to the injured striatum. Moreover, we observed an increase in axonal density in the cortex area by anterograde BDA tracer in the treated group compared with the control animals $(394.61 \pm 51.08$ vs. $1647.98 \pm 43.56$, respectively; $p<.05)$ at 4 weeks after treatment (Figure 3(e)). 


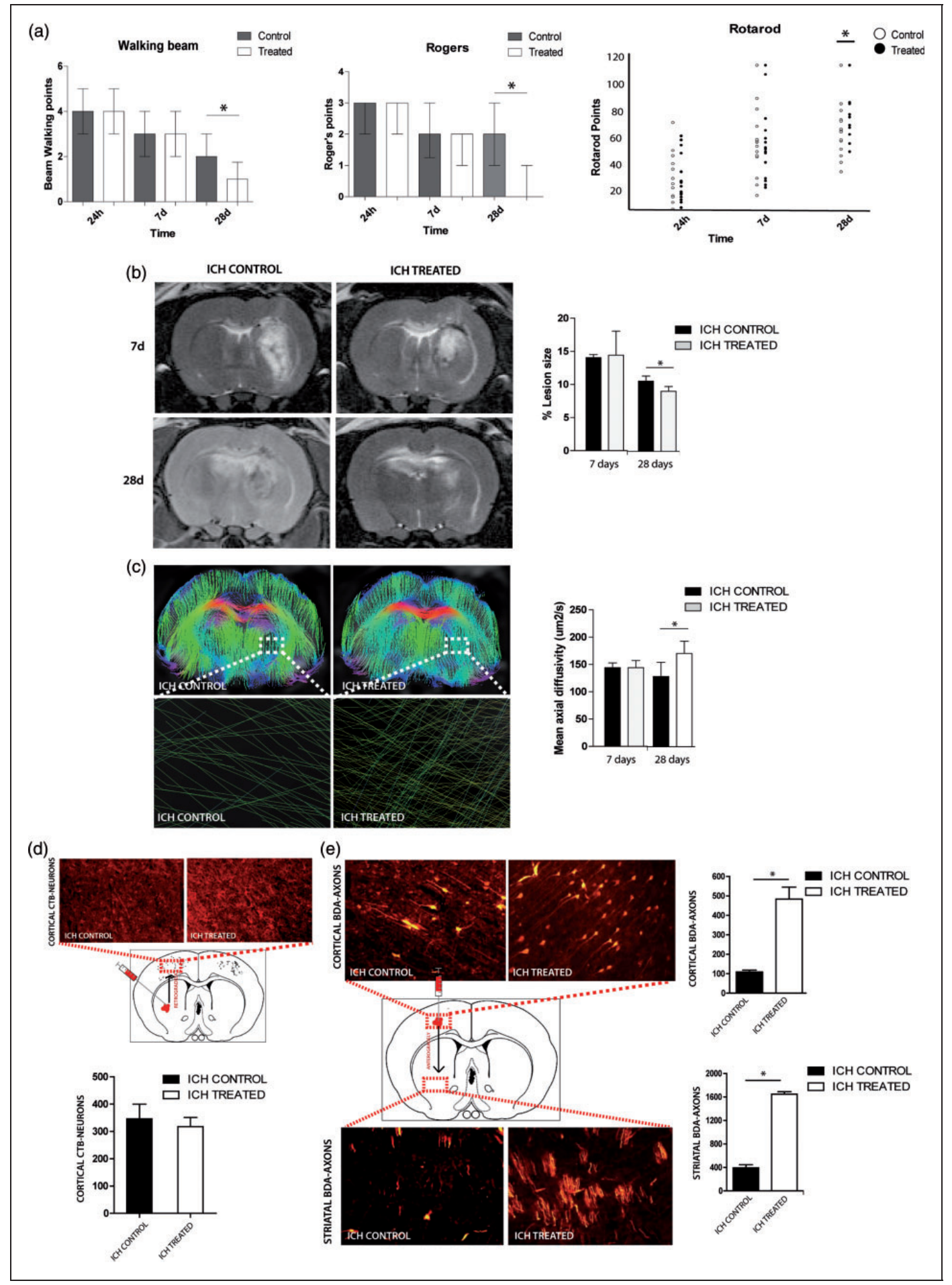

Figure 3. Improved functional outcome reduced infarct size and increased fiber tract and axonal sprouting after exosome treatment in intracerebral hemorrhage. (a) Beam Walking test performance (left) (data are shown as median \pm interquartile range, $* p<.05 ; \mathrm{n}=10$ animals per group), the Rogers test (middle) (data are shown as median \pm interquartile range, ${ }^{*} p<.05 ; n=10$ animals per group), and the Rotarod (right) (data are shown as bar graph, $\mathrm{n}=10$ animals per group) was improved at 28 days $(p<.05)$ in the treatment group compared with the controls $(p<.05)$. (b) Comparative image analysis of T2-weighted MRI at 7 and 28 days showed a progressive reduction in white matter infarct size of hemorrhagic stroke in the treated animals. (Data are shown as mean $\pm S D, * p<.05, n=4$ animals) (c) Comparative image analysis of tractography showed significant high tract connectivity in the treated animals compared with the controls at 28 days after treatment. Detail of tractography image in the lesion is displayed below, showing augmented connectivity of fiber tracts at 28 days. Quantitative analysis of MRI images and tractography. (Data are shown as mean $\pm S D, * p<.05, n=4$ animals). (d) Representative photomicrographs and quantification of cortical neuronal projections through the stroke site, labeled with the anatomical tracer cholera toxin subunit B (CTB). (e) Photomicrographs of cortical cells previously labeled with BDA and striatal axons from cortical cells labeled with biotinylated dextran amine (BDA) (left). Quantification of cortical BDA axons and striatal BDA axons (right). (Data are shown as mean \pm SEM, ${ }^{*} p<.05, \mathrm{n}=4$ animals, 10 sections in each animal per group). 


\section{Effect of exosome treatment on oligodendrocyte- associated marker expression}

Considering the expected time-related developmental steps of oligodendrocyte precursor cell (OPC) development, the levels of markers related to the first steps of oligodendrocyte progenitor cell development were analyzed in the lesion area 7 days after treatment (Figure 4).

CNP-ase appears to be one of the earliest indications of oligodendrocyte differentiation. Notably, using Western blot analysis, there was a significant increase in CNP-ase marker levels in the exosome-treated animals compared with the control animals at 7 days after treatment $(0.79 \pm 0.11$ vs. $0.61 \pm 0.03$, respectively; $p=.043$ ) (Figure 4(b) and (d)).

A2B5 is a cell surface ganglioside epitope expressed in OPC and its levels were higher in the exosome-treated animals compared with the control group at 7 days after treatment $(1.09 \pm 0.10$ vs. $0.82 \pm 0.13$, respectively; $p=.017$ ) (Figure 4(b) and (d)).

At 28 days after treatment, we analyzed one marker that was related to later developmental steps in mature oligodendrocytes (MOGs). After Western blot analysis, there were significantly higher levels of MOGs in the animals that received exosomes compared with the control group ( $1.60 \pm 0.22$ vs. $1.02 \pm 0.40$, respectively, $p=0.05$ ) (Figure 4(a) and (b)). All these results were

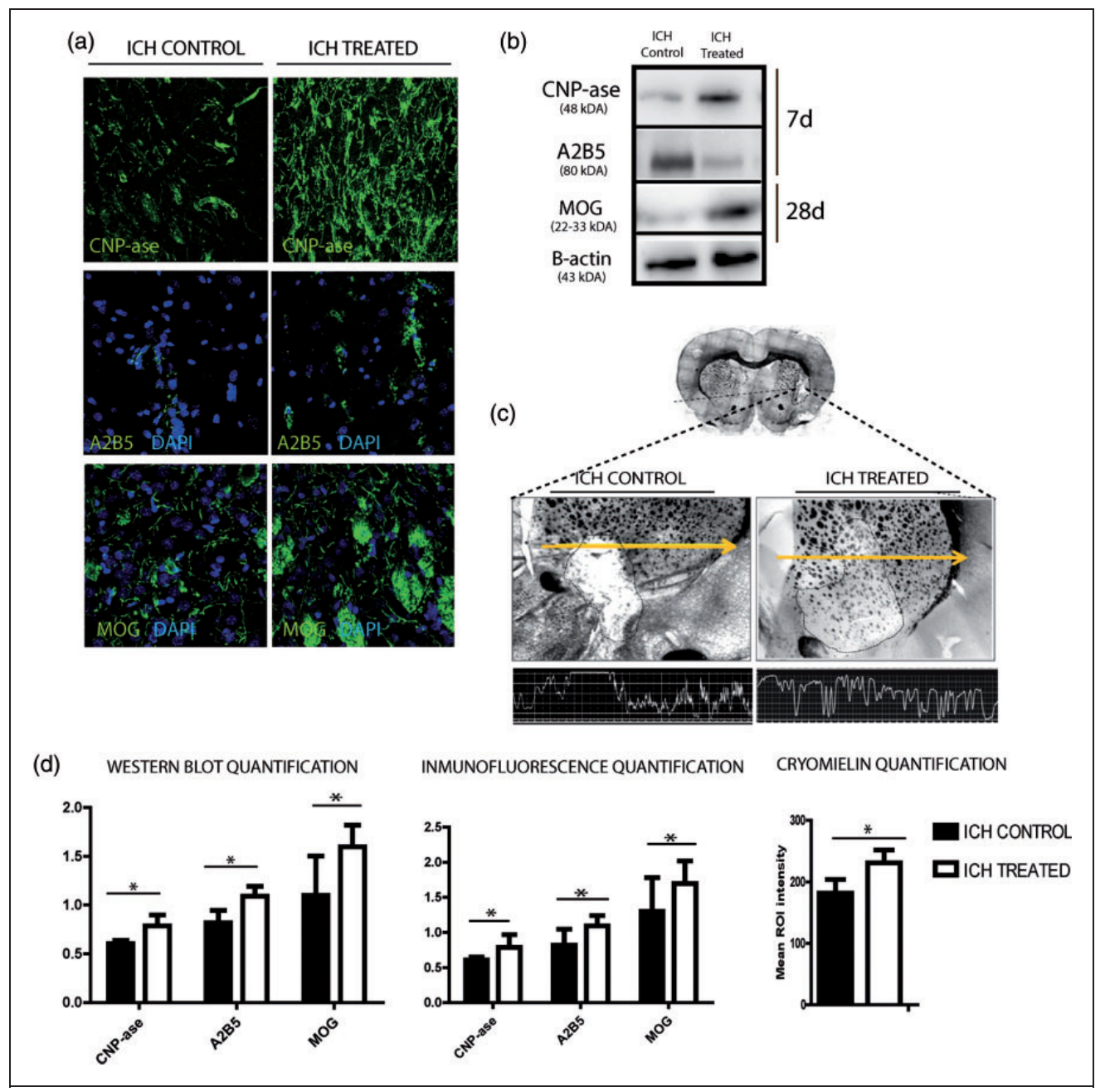

Figure 4. White matter-associated markers are enhanced after exosome therapy in subcortical stroke model. (a) Immunofluorescence images of white matter repair-associated markers (CNP-ase, A2B5 and MOG). (b) Western blot images. (c) Morphological study by CryoMyelin staining identified the zone of the lesion as an area of white matter injury located in the subcortical zone, showing restored myelinated axons in the exosome-treated animals. (d) Western blot and IF quantification of white matter repair-associated markers and quantification of mean ROI intensity of the CryoMyelin staining, respectively. Stroked line indicates ROI; yellow line indicates a representative longitudinal profile of pixel intensity. (Data are shown as mean \pm SEM, scale bars $=20 \mu \mathrm{m},{ }^{*} p<.05, \mathrm{n}=4$ animals, 5 sections in each animal per group, 10 different microscope fields). 
confirmed by immunofluorescence analysis (Figure 4(a) and (d)).

\section{Myelin restoration}

Morphological study using a Hito CryoMyelin staining kit identified the lesion zone in all the animals as a subcortical hemorrhage affecting the white matter, with more myelinated axons labeled in the exosometreated animals compared with the control group $(230.87 \pm 16.35$ vs. $181.72 \pm 22.18$, respectively, $p=.014)$ (Figure 4(c) and (d)).

\section{Proteomics analysis of the exosome}

The proteomics analysis of the exosome identified 2416 proteins (Figure 5) that are implicated in various cell functions, such as protein binding, macromolecular complex binding, heparin binding, protein complex binding, fibronectin binding, endopeptidase regulator activity and metal ion binding (Figure 5(a)). All the functions in detail are shown in Figure 5(c). Full proteomic data are provided in the supplementary data (S1). Interestingly, some of these proteins are involved in brain repair function, including synaptic transmission, neuronal differentiation from neural stem cells, angiogenesis, neuronal projections, neurite outgrowth, neurite fasciculation and axonal growth. The proteins related to these functions are synaptic vesicle membrane protein (VAT1), synaptotagmin-11 (SYT11), protein GDN, mitogen-activated protein kinase (M3K11), neuronal pentraxin-1 (NPTX1), vascular endothelial growth factor (VEGF165R), von Willebrand factor, neuropilin2 (NRP2), neurofascin (NFASC), neuronal cell adhesion molecule (NRCAM) and neurofilament (Figure 5(b)).

\section{Discussion}

In the current research for new therapeutic strategies to improve functional and cognitive deficits after stroke, axonal sprouting and growth, oligodendrocyte formation, tract connectivity and remyelination are necessary for brain repair processes. Thus, the present study employed, for the first time, an intravenous infusion of exosomes as a therapeutic strategy to repair white matter damage after intracerebral hemorrhage. In this study, after biodistribution analysis, the DiI marker was found in the brain and in the peripheral organs (liver, lung and spleen). The animals treated with exosomes showed better scores in functional tests, increased mean axial diffusivity by DTI-tractography and more axons, oligodendrocyte-associated marker expression and increased myelin staining. These results could support a role for MSC-derived exosomes in improving the repair of white matter in intracerebral hemorrhage.
Proteomics analysis of the exosomes identified 2416 proteins that are implicated in brain repair function, including synaptic transmission, neuronal differentiation from neural stem cells, angiogenesis, neuronal projections, neurite outgrowth, neurite fasciculation and axonal growth.

The high frequency of white matter damage in hemorrhage motivates the search for an effective therapy to enhance the mechanisms underlying the repair of damaged white matter (axons and myelin). Stem cell therapy is emerging as a viable repair therapy in stroke. $^{3,4,15}$ MSCs used as cell therapy might act as remote bioreactors leading to systemic remote effects on the central nervous system. ${ }^{24}$ Accordingly, the mechanisms by which the stem cells induce these functional improvements might be related to intercellular communication that can occur not only by direct cell-to-cell contact, but also by paracrine function by extracellular vesicles called exosomes through secreting factors in the extracellular environment. Thus, extracellular vesicles might partially explain the paracrine effects observed in stem cell-based therapeutic approaches and can play a beneficial role in restoring tissue and organ damage.

Exosomes purified from MSCs have generated greater interest based on their ability to reduce apoptosis/ necrosis in rodents after ischemic injury to the heart, ${ }^{25}$ brain, ${ }^{9}$ lung, ${ }^{26}$ liver ${ }^{27}$ and kidney. ${ }^{28}$ Some studies have shown that the GFP-tagged exosome-enriched extracellular particles released from MSCs intravenously administered to rats with stroke were transferred to adjacent astrocytes and neurons. ${ }^{11}$ In the present study, immunofluorescence for DiI-pre-labeled exosomes showed both intracellular and extracellular staining of DiI in the brain (area of injury) and colabeling with VEGF, NeuN, GFAP and Iba-1. DiI labeling was found not only in the brain, but also in peripheral organs such as the lung, liver and spleen at $24 \mathrm{~h}$ after treatment. Further investigation is warranted to determine whether these nano-sized exosomes are trapped in those organs and have remote effects on the brain.

Regarding functional recovery, previous studies by our group have demonstrated improvement in functional outcome after MSC administration following intracerebral hemorrhage. ${ }^{4}$ However, whether exosomes produced by MSCs had this function, mirroring that of their parent cells was still unknown. The present study demonstrated that rats receiving exosomes generated by MSCs showed better scores in functional tests in intracerebral hemorrhage 28 days after treatment. This result agrees with some studies that demonstrated systemic administration of MSC-generated exosomes significantly improved functional recovery in other experimental animal models of stroke (ischemia) ) $^{7,9,29}$ and traumatic brain injury. ${ }^{30}$ 

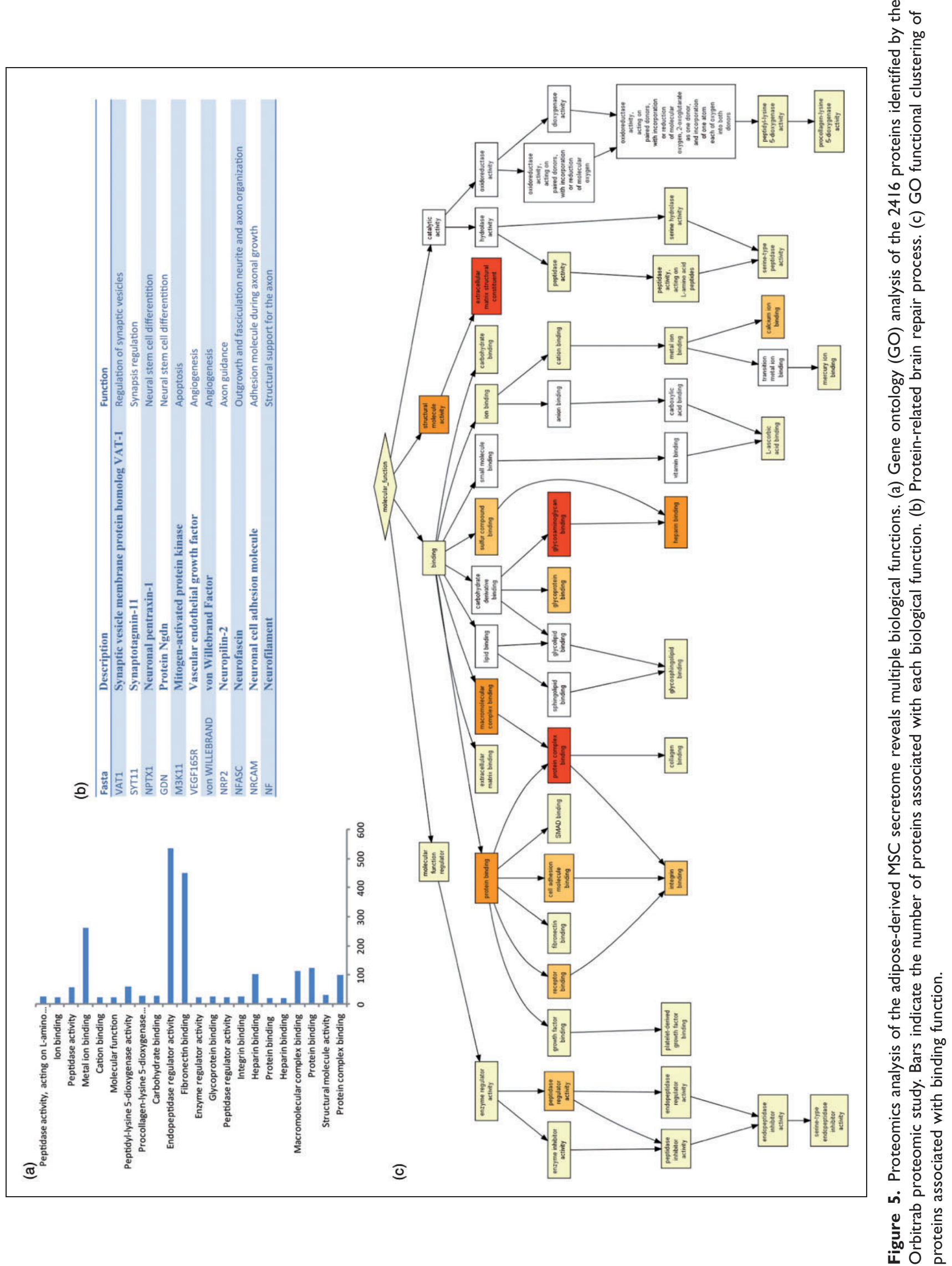
The mechanisms by which exosomes act are still unknown; further investigation is required. Recent studies demonstrate that intravenous administration of cellfree MSC-generated exosomes enhances neurovascular plasticity and neurite remodeling in rats after stroke. ${ }^{9}$ In our study, neurons were anterogradely labeled by a BDA injection into the cortex three weeks after stroke to map the connections that were growing after treatment. A statistically significant high number of BDA-labeled axons were shown in the striatum of the treated group compared with the control animals. These results might indicate that exosome treatment produces a significant axonal sprouting response from the ipsilateral cortex to the injured striatum. All these results agree with those obtained analyzing fiber tract integrity by DTI tractography. The animals receiving exosomes showed higher mean axial diffusivity compared with controls. These results could indicate that tract thickness was recovered at 28 days after exosome administration. These results suggest that good functional recovery at 28 days could be related to axonal sprouting white matter thickness (width, breadth, depth) and restoration of tract connectivity in the exosome-treated animals. For this reason, exosome administration could be involved in the process by which restructured axons, which had previously been compromised and demyelinated, recover not only their proper structure but also tract connectivity. These results are consistent with previous data showing neurite remodeling in the cortical ischemic boundary zone after other types of stroke. ${ }^{9}$

After a subcortical stroke, not only axonal and tract restoration but also oligodendrocyte and myelin formation are involved in a correct brain repair process. In this type of subcortical stroke, myelin injury activates the OPCs distributed throughout the white matter, which proliferate and migrate to the site of damage where they subsequently mature into myelinating oligodendrocytes that ensheath axons to form the myelin membrane. ${ }^{31}$ To elucidate whether exosome administration also acts on oligodendrocytes and shattered white matter fibers, we measured oligodendrocyte-associated markers in both the treated and the control animals. Related to the first steps of the genesis and migration of OPCs, markers such as A2B5 (a characteristic OPC marker) and CNP-ase (a marker related to immature oligodendrocytes) were studied in the lesion area at $7 \mathrm{~d}$ after treatment. Markers related to white matter differentiation and myelin fiber maturation such as MOGs were analyzed at 28 days.

The best-characterized oligodendroglial progenitor marker is A2B5. ${ }^{32}$ This molecule is a cell surface ganglioside expressed on developing oligodendroglial progenitors. In our study, the levels of the A2B5 marker were higher in the cell-free exosomes generated by the MSC-treated animals at 7 days than in the control group. This observation agrees with a study describing the levels of CNP-ase. The presence of CNP-ase appears to be one of the earliest events in oligodendrocyte differentiation. ${ }^{33}$ The exosome-treated animals showed higher levels of CNP-ase than the control group. Regarding the oligodendrocyte maturationassociated proteins, the levels of MOG markers were found to be elevated in the exosome-treated animals compared with the control animals at 28 days.

Not only are axons important in the process of white matter repair, but also the myelin sheath, which allows the transmission of nerve impulses over relatively long distances; a process necessary for whole brain function. A cryomyelin study showed an increase in myelination in those animals that received treatment with exosomes compared with the control group. What is unknown is whether the increased myelination was because more axons were being myelinated or whether there was an overall increase in myelin thickness.

Based on the results obtained in this study, we can conclude that exosomes enhance the natural repair processes of the brain after injury. However, the mechanisms underlying these repair processes are still unknown, given the identity of which components of the exosomes' proteome are responsible for this effect remains elusive. Our proteomics analysis identified 2416 proteins; some of these proteins are associated with several brain functions, such as synaptic transmission (VAT1, SYT11, NPTX1), neuronal differentiation from neural stem cells (GDN, NPTX1), apoptosis (M3K11, NPTX1), angiogenesis (VEGF165R, von Willebrand factor), neuronal projections (NRP2), neurite outgrowth, neurite fasciculation and axonal growth (NFASC, NRCAM, NF). All these functions are associated with brain repair after stroke. These results agree with other studies that revealed exosomes from MSCs reduced inflammation and increased cell proliferation during tissue repair following other types of stroke in rats, promoting angiogenesis, neurogenesis, neurite outgrowth and recovery. ${ }^{9,34}$

The gene ontology analysis revealed that protein binding, macromolecular complex binding, heparin binding, protein complex binding, fibronectin binding, endopeptidase regulator activity and metal ion binding were the most important functions of the identified proteins. Interestingly, growth factor activity was not the primary represented function in the cell secretome, indicating that other functions are also relevant. Our findings suggest that the identified proteins could contribute to improved functional recovery after exosome administration. When the specific molecules necessary for a therapeutic effect are known, selective manipulation of the expression of those molecules might lead to an enhancement of the therapeutic efficiency of isolated exosomes.

Finally, the therapeutic use of exosomes can be advantageous because of their ability to reduce the 
high production costs of stem cell treatment. For therapeutic treatment, exosomes can be frozen and stored in hospital freezers, as needed. For example, this treatment can be immediately administered to the patient upon admission and diagnosis; thus, treatment can be administered in the acute phase of stroke. Moreover, using exosomes as alternative therapy for stroke would decrease the cost of treatment.

In conclusion, in the current research, we have demonstrated for the first time that rats receiving exosomes derived from adipose-derived MSCs as a therapeutic strategy showed improved functional recovery after intracerebral hemorrhage, more axons, a higher expression of oligodendrocyte formation markers, higher tract connectivity and greater myelin staining, which are necessary for brain repair processes. Proteomic analyses identified 2416 proteins, some of these are associated with several brain functions. Thus, exosome treatment could be a heterogeneous process by nature, with many factors that can influence brain plasticity in an adaptable and versatile way; however, future work will be necessary to confirm their implications in intracerebral hemorrhage.

\section{Limitation of the study}

A limitation of the present study is that only males were used for the experiments. Following the STAIR recommendations, future studies should be performed in both males and females in order to translate preclinical studies to the clinic.

Another limitation of this study is regarding $\mathrm{ICH}$ induced by collagenase IV; the levels of this enzyme are significantly greater than the levels of endogenous collagenase in clinical ICH, which might not allow this model to faithfully emulate the pathological alterations involved in ICH in the clinical setting. The disadvantages of this model are related to bacterial collagenase's ability to introduce an inflammatory reaction. Moreover, in opposition to ICH in patients in which the solid hematoma is a result of bleeding from the arterial source in brain tissue, bleeding in the collagenase model is diffuse and results from rupture of small blood vessels around the site of collagenase injection. ${ }^{35}$ However, any experimental animal model reproduces completely the clinical disease. Most of the studies based on experimental animal models of hemorrhage use collagenase Type IV in order to induce ICH.

\section{Funding}

The author(s) disclosed receipt of the following financial support for the research, authorship, and/or publication of this article: This study has been partially supported by grants from PS15/01318 and INVICTUS (RD12/0014) (Spanish Neurovascular Network), Miguel Servet (CP15/00069 to
María Gutiérrez-Fernández) and a Sara Borrell postdoctoral fellowship (CD12/00706, to Laura Otero-Ortega) from the Research Institute Carlos III, the Spanish Ministry of Science and Innovation and the European Regional Development Fund. This study was also co-funded within the Roche Farma project. The funders had no role in study design, data collection and analysis, decision to publish or preparation of the manuscript.

\section{Acknowledgments}

We greatly appreciate the support of ServingMed.com for editing assistance. We also thank Rosario Madero from the Department of Statistics for technical assistance. We would also like to thank Prof. Carmichael for his supervision of Laura Otero-Ortega's work in his laboratory regarding neuronal tracer experiments.

\section{Declaration of conflicting interests}

The author(s) declared no potential conflicts of interest with respect to the research, authorship, and/or publication of this article.

\section{Authors' contributions}

LOO: Collection and/or assembly of data, manuscript writing, data analysis and interpretation. MCGF: Collection and/ or assembly of data. FLG: Collection and/or assembly of data. BRF: Collection and/or assembly of data. EGM: Collection and/or assembly of data. JAL: Collection and/or assembly of data. JV: Collection and/or assembly of data. EDT: Conception and design, data analysis and interpretation, final approval of the manuscript. MGF: Conception and design, data analysis and interpretation, final approval of the manuscript.

\section{Supplementary material}

Supplementary material for this paper can be found at the journal website: http://journals.sagepub.com/home/jcb

\section{References}

1. Matute C, Domercq M, Pérez-Samartin A, et al. Protecting white matter from stroke injury. Stroke 2013; 44: 1204-1211.

2. Zhang R, Chopp M and Zhang ZG. Oligodendrogenesis after cerebral ischemia. Front Cell Neurosci 2013; 7: 201.

3. Otero L, Bonilla C, Aguayo C, et al. Intralesional administration of allogeneic bone marrow stromal cells reduces functional deficits after intracerebral hemorrhage. Histol Histopathol 2010; 25: 453-461.

4. Otero L, Zurita M, Bonilla C, et al. Allogeneic bone marrow stromal cell transplantation after cerebral hemorrhage achieves cell transdifferentiation and modulates endogenous neurogenesis. Cytotherapy 2012; 14: 34-44.

5. Otero-Ortega L, Gutiérrez-Fernández M, Ramos-Cejudo $\mathrm{J}$, et al. White matter injury restoration after stem cell administration in subcortical ischemic stroke. Stem Cell Res Ther 2015; 6: 121. 
6. Gutiérrez-Fernández M, Rodríguez-Frutos B, Alvarez-Grech J, et al. Functional recovery after hematic administration of allogenic mesenchymal stem cells in acute ischemic stroke in rats. Neuroscience 2011; 175: 394-405.

7. Doeppner TR, Herz J, Görgens A, et al. Extracellular vesicles improve post-stroke neuroregeneration and prevent postischemic immunosuppression. Stem Cells Transl Med 2015; 4: 1131-1143.

8. Paschon V, Takada SH, Ikebara JM, et al. Interplay between exosome, microRNAs and Toll-like receptors in brain disorders. Mol Neurobiol 2016; 53: 2016-2028.

9. Xin H, Li Y, Cui Y, et al. Systemic administration of exosome released from mesenchymal stromal cells promote functional recovery and neurovascular plasticity after stroke in rats. J Cereb Blood Flow Metab 2013; 33: 1711-1715.

10. Théry C, Zitvogel L and Amigorena S. Exosome: composition, biogenesis and function. Nat Rev Immunol 2002; 2: 569-579.

11. Xin H, Li Y, Liu Z, et al. MiR-133b promotes neural plasticity and functional recovery after treatment of stroke with multipotent mesenchymal stromal cells in rats via transfer of exosomes-enriched extracellular particles. Stem Cells 2013; 31: 2737-2746.

12. Stroke Therapy Academic Industry Roundtable (STAIR). Recommendations for standards regarding preclinical neuroprotective and restorative drug development. Stroke 1999; 12: 2752-2758.

13. Fisher M, Feuerstein G, Howells DW, et al. Update of the stroke therapy academic industry roundtable preclinical recommendations. Stroke 2009; 40: 2244-2250.

14. Kilkenny C, Browne W, Cuthill IC, et al. Animal research: reporting in vivo experiments: the ARRIVE guidelines. Br J Pharmacol 2010; 160: 1577-1579.

15. Otero L, Zurita M, Bonilla C, et al. Late transplantation of allogeneic bone marrow stromal cells improves neurologic deficits subsequent to intracerebral hemorrhage. Cytotherapy 2011; 13: 562-571.

16. Kumar D, Gupta D, Shankar S, et al. Biomolecular characterization of exosomes released from cancer stem cells: possible implications for biomarker and treatment of cancer. Oncotarget 2015; 6: 3280-3291.

17. Ramos-Cejudo J, Gutiérrez-Fernández M, Otero-Ortega $\mathrm{L}$, et al. Brain-derived neurotrophic factor administration mediated oligodendrocyte differentiation and myelin formation in subcortical ischemic stroke. Stroke 2015; 46: 221-228.

18. Rogers DC, Campbell CA, Stretton JL, et al. Correlation between motor impairment and infarct volume after permanent and transient middle cerebral artery occlusion in the rat. Stroke 1997; 28: 2060-2065.

19. Rodríguez-Frutos B, Otero-Ortega L, Ramos-Cejudo J, et al. Enhanced brain-derived neurotrophic factor delivery by ultrasound and microbubbles promotes white matter repair after stroke. Biomaterials 2016; 100: 41-52.

20. Barratt HE, Lanman TA and Carmichael ST. Mouse intracerebral hemorrhage models produce different degrees of initial and delayed damage, axonal sprouting, and recovery. J Cereb Blood Flow Metab 2014; 34: 1463-1471.

21. Wiśniewski JR, Zougman A, Nagaraj N, et al. Universal sample preparation method for proteome analysis. Nat Methods 2009; 5: 359-362.

22. Navarro P, Trevisan-Herraz M, Bonzon-Kulichenko E, et al. General Statistical framework for quantitative proteomics by stable isotope labeling. J Proteome Res 2014; 3: 1234-1247.

23. Eden E, Navon R, Steinfeld I, et al. GOrilla: a tool for discovery and visualization of enriched GO terms in ranked gene lists. BMC Bioinformatics 2009; 10: 48.

24. Walker PA, Shah SK, Jimenez F, et al. Bone marrowderived stromal cell therapy for traumatic brain injury is neuroprotective via stimulation of non-neurologic organ systems. Surgery 2012; 152: 790-793.

25. Lai RC, Arslan F, Lee MM, et al. Exosomes secreted by MSC reduces myocardial ischemia/reperfusion injury. Stem Cell Res 2010; 4: 214-222.

26. Lee C, Mitsialis SA, Aslam M, et al. Exosome mediate the cytoprotective action of mesenchymal stromal cells on hypoxia-induced pulmonary hypertension. Circulation 2012; 126: 2601-2611.

27. Kanazawa H, Fujimoto $\mathrm{Y}$, Teratani $\mathrm{T}$, et al. Bone marrow-derived mesenchymal stem cells ameliorate hepatic ischemia reperfusion injury in a rat model. PLoS One 2011; 6: e19195.

28. Gatti S, Bruno S, Deregibus MC, et al. Microvesicles derived from human adult mesenchymal stem cells protect against ischaemia-reperfusion-induced acute and chronic kidney injury. Nephrol Dial Transplant 2011; 26: 1474-1483.

29. Zhang ZG and Chopp M. Exosome in stroke pathogenesis and therapy. J Clin Invest 2016; 126: 1190-1197.

30. Zhang Y, Chopp M, Meng Y, et al. Effect of exosome derived from multipluripotent mesenchymal stromal cells on functional recovery and neurovascular plasticity in rats after traumatic brain injury. J Neurosurg 2015; 122 : 856-867.

31. Sozmen EG, Kolekar A, Havton LA, et al. A white matter stroke model in the mouse: axonal damage, progenitor responses and MRI correlates. J Neurosci Meth 2009; 180: 261-272.

32. Back SA, Riddle A and McClure MM. Maturationdependent vulnerability of perinatal white matter in premature birth. Stroke 2007; 38: 724-730.

33. Baracskay KL, Kidd GJ, Miller RH, et al. NG2-positive cells generate A2B5-positive oligodendrocyte precursor cells. Glia 2007; 55: 1001-1010.

34. Xin H, Li Y, Buller B, et al. Exosomes-mediated transfer of miR-133b from multipotent mesenchymal stromal cells to neural cells contributes to neurite outgrowth. Stem Cells 2012; 30: 1556-1564.

35. Manaenko A, Chen H, Zhang JH, et al. Comparison of different preclinical models of intracerebral hemorrhage. Acta Neurochir Suppl 2011; 111: 9-14. 